\title{
Entwicklung eines Low-Cost PM Sensorenclusters zur Untersuchung des Abscheideverhaltens eines Nassabscheiders
}

\author{
Felix Reinke ${ }^{1}$, Michael Novosel2, Jörg Meyer ${ }^{1}$ und Achim Dittler ${ }^{1}$ \\ ${ }^{1}$ Institut für Mechanische Verfahrenstechnik und Mechanik (MVM), Karlsruher Institut für Technologie (KIT), \\ Karlsruhe, Deutschland \\ ${ }^{2}$ ESTA Apparatebau GmbH \& Co. KG, Senden, Deutschland \\ Kontakt: felix.reinke@kit.edu
}

\section{Einleitung}

Durch Fortschritte der metallischen Zerspanungsverfahren (Schleifen, Bürsten, Polieren etc.) kommt es zur Entstehung von submikronen Partikelstäuben, deren Abscheidung immer komplexer wird. Treten alveolengängige Stäube auf (vornehmlich $\mathrm{PM}_{1}$ und $\mathrm{PM}_{2,5}$ Fraktion), bedarf es einer im Prozess integrierten Abscheidung, welche jedoch mit einem zusätzlichen Druckverlust des Gesamtsystems einhergeht und den notwendigen Energieeintrag erhöht. Aus diesem Grund sind neuartige Technologien erforderlich, welche bei minimalem Energieeintrag eine hohe Abscheideeffizienz (im Bereich der submikronen Partikeln) erreichen. Eine Möglichkeit können hierbei nassabscheidende Verfahren mit innovativen 3D-gedruckten Düsengeometrien zur Dispergierung der Waschflüssigkeit bieten.

Zur kostengünstigen Beurteilung der Abscheideeffizienz der Partikelfraktionen und zum besseren Verständnis von lokalen Partikelkonzentrationsniveaus können sogenannte Low-Cost PM-Sensoren lokal in den Nassabscheider eingebaut werden. Diese haben sich bereits im Anwendungsfeld der Immissionsmessung und Emmisionscharakterisierung zur Erfassung der zeitlichen und lokalen Partikelkonzentration etabliert. [1] Aufgrund der vorherrschenden Betriebsbedingungen bei der Nassabscheidung kann allerdings die qualitative Aussagekraft der Sensordaten durch zeitlich abweichende Quereinflüsse (z.B: relative Luftfeuchte) beeinflusst werden. [2]

Im Zuge dieses Beitrags soll die Methodik zur Entwicklung eines Sensorenclusters Low-Cost PM-Sensoren zur Untersuchung des lokal aufgelösten Abscheideverhaltens in einem Nassabscheider vorgestellt werden. Mit Hilfe der aus dieser Versuchsmethodik gewonnen experimentellen Daten soll zukünftig ein adaptiver Nassabscheider entwickelt werden, welcher sich entsprechend der vorherrschenden Randbedingungen (z.B. Partikelkonzentration, Strömungsverhältnisse) anpasst und somit bei multiplen Betriebspunkten fähig ist, im energieeffizienten Optimum bei maximaler Abscheideleistung betrieben zu werden.

Hierzu soll zunächst anhand von theoretischen Grundlagen die zu untersuchende Aerosolstreulichtpartikelmesstechnik erläutert werden. Anschließend folgt eine Vorstellung der verwendeten
Partikelmesstechnik. Daraufhin soll die grundlegende Vorgehensweise zur systematischen Charakterisierung der qualitativen Aussagekraft der Sensordaten vorgestellt werden.

\section{Grundlagen und Materialien}

\section{Nassabscheider}

Grundsätzlich basieren Abscheider auf dem Prinzip, dass die Bahnkurven, auf denen sich die Partikeln im Fluid bewegen, zu Bereichen oder Flächen führen müssen, von denen die Partikeln durch die kontinuierliche Phase nicht mehr wegbefördert werden können. Diese Bereiche bzw. Flächen werden als Kollektoren bezeichnet. In der Nassabscheidung dient die Tropfenoberfläche als Kollektor. Sobald eine Partikel auf einen Tropfen trifft, kann nach Weber [3] angenommen werden, dass diese haften bleiben. Das dadurch gebildete Partikel-Tropfen-Agglomerat lässt sich, aufgrund der erhöhten Masse im Vergleich zur Partikel, besser mit Hilfe von beispielsweise trägheitsbasierten Abscheidemechanismen aus dem Gasstrom entfernen. [4]

Die in einem Nassabscheider ablaufenden Vorgänge können in vier Bereiche eingeteilt werden:

- Dispergierung der Waschflüssigkeit im Aerosolstrom

- Kollision der dispersen Phase mit den Tropfen

- Haftung der Partikeln an der Tropfenoberfläche

- Abscheidung der partikelbeladenen Tropfen stromabwärts

Nassabscheider unterscheiden sich zum einen in der Art der Tropfenerzeugung und zum anderen in der Art der Vermischung des Aerosols mit der Waschflüssigkeit. Beim zu untersuchenden Nassabscheider zerstäubt die Waschflüssigkeit in der Kontaktzone durch Ansaugung und Überströmung der Flüssigkeit.

\section{Messtechnische Grundlagen}

Zur online Messung von Partikelkonzentrationen in einem Gasstrom finden unter anderem optische Verfahren Anwendung. [5]

Dabei erfolgt eine Unterscheidung in abbildende Verfahren und Streulichtverfahren. Da die im Rahmen dieser Studie verwendete Partikelmesstechnik ausschließlich auf dem Streulichtverfahren basiert findet nachstehend lediglich eine Betrachtung dieses Verfahrens statt. 
Das Messprinzip der Streulichtmesstechnik basiert auf der Streuung einer Lichtwelle mit der Wellenlänge $\lambda$ an einer kugelförmigen Partikel mit dem Durchmesser $\mathrm{d}$ und dem Brechungsindex $\mathrm{n}$. Aufgrund von Beugung, Reflexion und Brechung findet eine Richtungsänderung der Ausbreitung des Lichts statt. Abbildung 1 stellt die Lichtstreuung an einer Einzelpartikel dar. Zusätzlich ist sowohl der Streuwinkel $\theta$, als auch der Polarisationswinkel $\phi$ aufgetragen. [6]



Abbildung 1 - Schematische Darstellung der Streuung einer Lichtwelle an einer Partikel. $\lambda$ steht hierbei für die Lichtwellenlänge, $n$ für den Brechungsindex, $d$ für den Partikeldurchmesser, $\theta$ für den Detektionswinkel des Streulichts und $\phi$ für den Polarisationswinkel der gestreuten Lichtwelle. Nach [4].

Aus Abbildung 1 ist erkennbar, dass die Lichtstreueffekte Funktionen der Partikeleigenschaften, der Wellenlänge des Lichtes und dessen Verhältnis zueinander sowie des Streuwinkels zwischen Beobachter und Beleuchtungsrichtung sind. Zur Beschreibung des Verhältnisses zwischen Partikelgröße und Wellenlänge wird ein Streuparameter $\alpha$ eingeführt:

$$
\alpha=\pi d / \lambda
$$

Die Intensität I des am Einzelpartikel gestreuten Lichts ergibt sich demnach aus der Intensität $\mathrm{I}_{0}$ des auf die Partikel treffenden Lichtes, dem Polarisationswinkel, dem Detektionswinkel, dem Brechungsindex $m$ und dem Streuparameter $\alpha$. Die relative Streulichtintensität $I^{*}$ stellt sich wie folgt dar:

$$
I^{*}=I / I_{0}=f(\phi, \theta, n, \alpha) \text {. }
$$

Mittels des Streuparameter $\alpha$ lassen sich die theoretischen Ansätze zur Beschreibung der Partikelgrößen- und Winkelabhängigkeit der Streulichtintensität in folgende drei Bereiche aufteilen [7]:

- $\quad \alpha<1$; Rayleigh-Bereich

- $\alpha \approx 1$; Mie-Bereich

- $\quad \alpha \gg>1$; Fraunhofer-Bereich

In Abbildung 2 ist die relative Streulichtintensität $\mathrm{I}^{*}$ über den Streuparameter $\alpha$ für monochromatisches Licht und einem festen Streuwinkel aufgetragen und die unterschiedlichen Bereiche des Streuparameters $\alpha$ gekennzeichnet.



Abbildung 2 - Schematische Darstellung des Zusammenhangs der Streulichtintensität über den Größenparameter $\alpha$. Zusätzlich sind die drei unterschiedlichen Bereiche der Streulichtintensität $(\alpha<<1 ; \alpha \approx 1 ; \alpha>>1)$ und deren Proportionalität zum Partikeldurchmesser aufgetragen. Nach [7].

Die in Abbildung 2 dargestellte Abhängigkeit der relativen Streulichtintensität weist im Rayleigh Bereich $(\alpha<<1)$ eine Abhängigkeit vom Partikeldurchmesser in der sechsten Potenz auf. Im Bereich der geometrischen Optik $(\alpha>>1)$, besteht eine quadratische Abhängigkeit der Streulichtintensität zum Partikeldurchmesser. Im Mie-Bereich $(\alpha \approx 1)$ sind starke Schwankungen zu erkennen und eine eindeutige Zuordnung der Streulichtintensität zur Partikelgröße ist allgemein nicht möglich. Das bedeutet, dass in der messtechnischen Anwendung einer gemessenen Lichtintensität mehr als eine Partikelgröße zugeordnet werden kann.

Zur Partikelmessung im Mie-Bereich besteht die Möglichkeit, unter Verwendung von weißem Licht mit $90^{\circ}$ Streulichtdetektion, eine Glättung der Kurve (vgl. Abbildung 2) zu erreichen und somit eine eindeutige Zuordenbarkeit zwischen gemessener relativer Streulichtintensität und Partikelgröße herzustellen. Eine weitere Möglichkeit besteht darin mittels monochromatischen Lichts mit $90^{\circ}$-Streulichtdetektion und einer Approximationsfunktion einen angenäherten Zusammenhang zu erhalten. [8]

Zur Ermittlung der Partikelgröße mittels Streulichtverfahren ist das Reflexionsverhalten und die Brechung der Wellen beim Auftreffen einer Phasengrenzfläche relevant. Dieses Verhalten wird durch den Brechungsindex $m$ beschrieben. Der Brechungsindex setzt sich aus einem Real- und einem Imaginärteil zusammen. Der Realteil ist hierbei der maßgebliche Parameter für die Lichtstreuung und der Imaginärteil beschreibt das Reflexionsverhalten. Unter der Annahme, dass das Reflexionsverhalten bei den zu untersuchenden Stoffen vernachlässigbar ist, ergibt sich der Brechungsindex als Verhältnis von der Lichtgeschwindigkeit im Vakuum c zur Lichtgeschwindigkeit im Partikel VP zu

$$
n=c / v_{P} .
$$




\section{Aufbau Streulichtmesstechnik}

Der schematische Aufbau eines Streulichtpartikelzählers ist in Abbildung 3 dargestellt. Darauf ist eine exemplarische Messkammer, eine Lichtquelle, die Aerosolzufuhr und Absaugung, sowie die Lichtfalle, ein Verstärker und ein Detektor abgebildet. Des Weiteren ist das Messvolumen gekennzeichnet. Über die Aerosolzufuhr, wird das zu untersuchende Aerosol in definierter Menge in die Messkammer bzw. in das Messvolumen gesaugt. Beim Auftreffen des Lichtstrahls mit einer Partikel wird dieser gestreut. Daraufhin findet eine messtechnische Detektion der Streuung statt. Anschließend wird die detektierte Streuung in ein elektrisches Signal umgewandelt und verstärkt. [9]

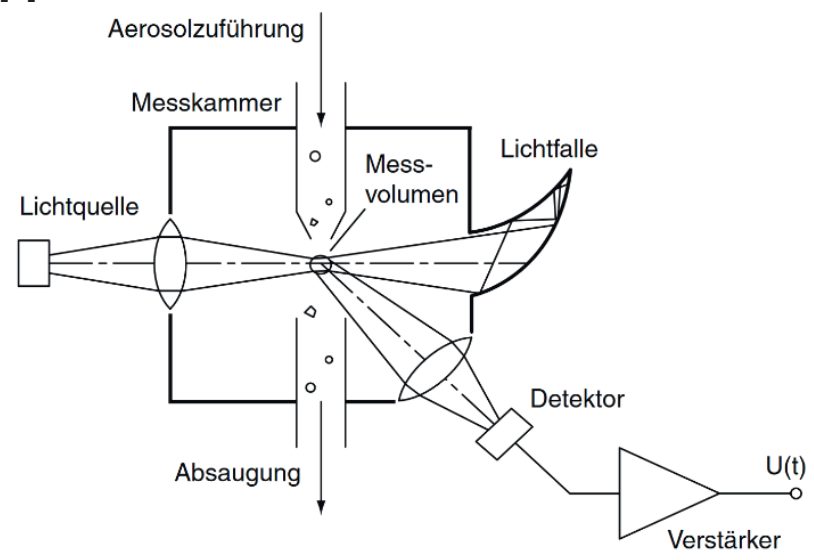

Abbildung 3 - Schematischer Aufbau eines Streulichtpartikelzählers. Dargestellt ist die Aerosolzuführung und -absaugung, eine Lichtquelle mit Detektor und Signalverstärker, sowie das Messvolumen und die Lichtfalle. [7]

Zur Vermeidung von Koinzidenzfehlern sollte bei einer Messung mit Streulichtpartikelzählern darauf geachtet werden, dass sich immer nur eine Partikel im Messvolumen während der Signalverarbeitung befindet. Bei Mehrfachdetektion von Partikeln werden mehrere Partikeln als ein großes Partikel erfasst, da keine eindeutige Zuordnung des Signals zur Partikelgröße stattfindet. Dadurch kommt es zu einer Fehlinterpretation der Partikelkonzentration und -größe. Koinzidenzfehler können durch die Wahl eines geeigneten Messvolumens reduziert werden. Dabei gilt, je kleiner das Messvolumen, desto höher ist die Partikelanzahlkonzentrationen die mit dem Gerät gemessen werden kann. [7]

Zusätzlich zu den Koinzidenzfehlern kann es zu Randzonenfehlern im Messvolumen kommen. Diese treten auf, wenn eine Partikel am Rand des Messvolumens eine deutlich geringere Streulichtintensität aufweist, wie eine Partikel selber Art im Zentrum des Messvolumens.

\section{Aerosolmesstechnik}

Die in diesem Beitrag verwendete Aerosolmesstechnik basiert auf dem Streulichtprinzip. Dabei wird ein HC-Sensor (High-Cost Sensor) als etablierte
Referenzpartikelmesstechnik verwendet, während die Tauglichkeit eines Low-Cost PM-Sensors (LCSensor) für die Anwendung der Emissionsüberwachung im Bereich der Nassabscheidung untersucht werden soll. Die von den Herstellern angegeben technischen Daten des jeweiligen Sensors sind auszugsweise in Tabelle 1 dargestellt. Hierbei fallen zunächst die Unterschiede bzgl. der Abmessungen und des Gewichts der Sensoren auf. Des Weiteren besteht die Möglichkeit bei der verwendeten Referenzmesstechnik (HC-Sensor) größenaufgelöst zu messen, während beim LC-Sensor lediglich fünf Größenkanäle zur Verfügung stehen. Außerdem ist der Anwendungsbereich der Referenzmesstechnik aufgrund einer beheizbaren Zulaufstrecke und variablen Einstellmöglichkeiten bzgl. beispielsweise Strömungsgeschwindigkeiten zur Gewährleistung einer isokinetischen Probenahme deutlich größer. Diese Variabilität spiegelt sich auch in der Verarbeitbarkeit der Messdaten wieder. Beim LC-Sensor gibt es keine Möglichkeit partikelmaterialspezifische Eigenschaften wie den Brechungsindex oder die Partikeldichte zu variieren. Ein weiterer messtechnisch relevanter Unterschied ist die Verwendung der Lichtquelle. Der HC-Sensor verwendet eine Weißlichtquelle und der LC-Sensor monochromatisches Licht. Wie bereits im Abschnitt „Messtechnische Grundlagen“ erklärt, unterscheiden sich die beiden Lichtquellen in der Art der Zugänglichkeit des Zusammenhangs zwischen relativer Streulichtintensität und dem Mie-Größenparameter.

Tab. 1: Technische Daten der verwendeten Partikelmesstechnik

\begin{tabular}{|c|c|c|}
\hline Sensortyp & HC-Sensor & LC-Sensor \\
\hline $\mathrm{Maße} / \mathrm{mm}$ & $50 \times 250 \times 100$ & $41 \times 41 \times 12$ \\
\hline Gewicht / g & 2800 & 6 \\
\hline Messbereich / $\mu \mathrm{m}$ & $\begin{array}{c}0,2-40 \\
3 \text { Bereiche }\end{array}$ & $0,3-10$ \\
\hline Messgrößen & $\begin{array}{l}\text { Partikelgrößen- } \\
\text { verteilung, } \\
\text { Partikelanzahl }\end{array}$ & $\begin{array}{c}\mathrm{PM}_{1}, \mathrm{PM}_{2,5} \\
\mathrm{PM}_{4}, \mathrm{PM}_{10}, \\
\text { Partikelanzahl }\end{array}$ \\
\hline Größenkanäle & 64 bins & 5 bins \\
\hline $\begin{array}{l}\text { Max. Anzahlkonzentra- } \\
\text { tion } / \mathrm{cm}^{-3}\end{array}$ & $5 * 10^{5}$ & $3 * 10^{3}$ \\
\hline Betriebstemperatur $/{ }^{\circ} \mathrm{C}$ & $\leq 250$ & $-10-60$ \\
\hline Max. rel. Luftfeuchte & heizbar & $80 \%$ \\
\hline Brechungsindex & variabel & konstant \\
\hline Lichtquelle & Weißlicht & $\begin{array}{l}\text { Monochroma- } \\
\text { tisch }\end{array}$ \\
\hline Partikeldichte & variabel & konstant \\
\hline $\begin{array}{l}\text { Max. Strömungsge- } \\
\text { schwindigkeiten } / \mathrm{ms}^{-1}\end{array}$ & $\begin{array}{l}\text { variabel über } \\
\text { isokinetische } \\
\text { Probenahmen }\end{array}$ & 1 \\
\hline Aufzeichnungsrate / Hz & 1 & 1 \\
\hline Kosten $/ €$ & $\sim 30.000$ & $\sim 40$ \\
\hline
\end{tabular}

Zusätzlich gibt Sensor 1 ebenfalls an, ob bzw. wie hoch der Koinzidenzfehler der aktuellen Messung ist. Diese Möglichkeit bietet LC-Sensor 2 nicht. Wie hier 
mit Koinzidenzfehlern umgegangen wird ist nicht bekannt. Des Weiteren ist beim $\mathrm{HC}$-Sensor eine $\mathrm{T}$ Blende zur Verringerung von Randzonenfehlern verbaut. Auch hier ist keine Information zum Umgang mit Randzonenfehlern vom LC-Sensor verfügbar.

\section{Methodisches Vorgehen}

Im Folgenden Kapitel soll das methodische Vorgehen zur Entwicklung eines Low-Cost PM-Sensorenclusters zur Untersuchung des Abscheideverhaltens eines innovativen Nassabscheiders vorgestellt werden. Zu Beginn der Methodik wird Funktion der Versuchsanlage beschrieben. Anschließend wird auf die strömungsmechanische und thermodynamische Charakterisierung der zur Verfügung stehenden Messpositionen eingegangen. Daraufhin folgt zunächst die Überprüfung möglicher Abweichungen zwischen den LC-Sensoren. Anschließend soll die qualitative Aussagekraft der Messdaten der LC-Sensoren mithilfe von etablierter Referenzmesstechnik (HC-Sensor) an den zuvor definierten Messpositionen verglichen werden.

\section{Funktionsbeschreibung Versuchsanlage}

Abbildung 4 stellt schematisch ein Fließschema der Versuchsanlage mit den relevantesten Funktionszonen dar.

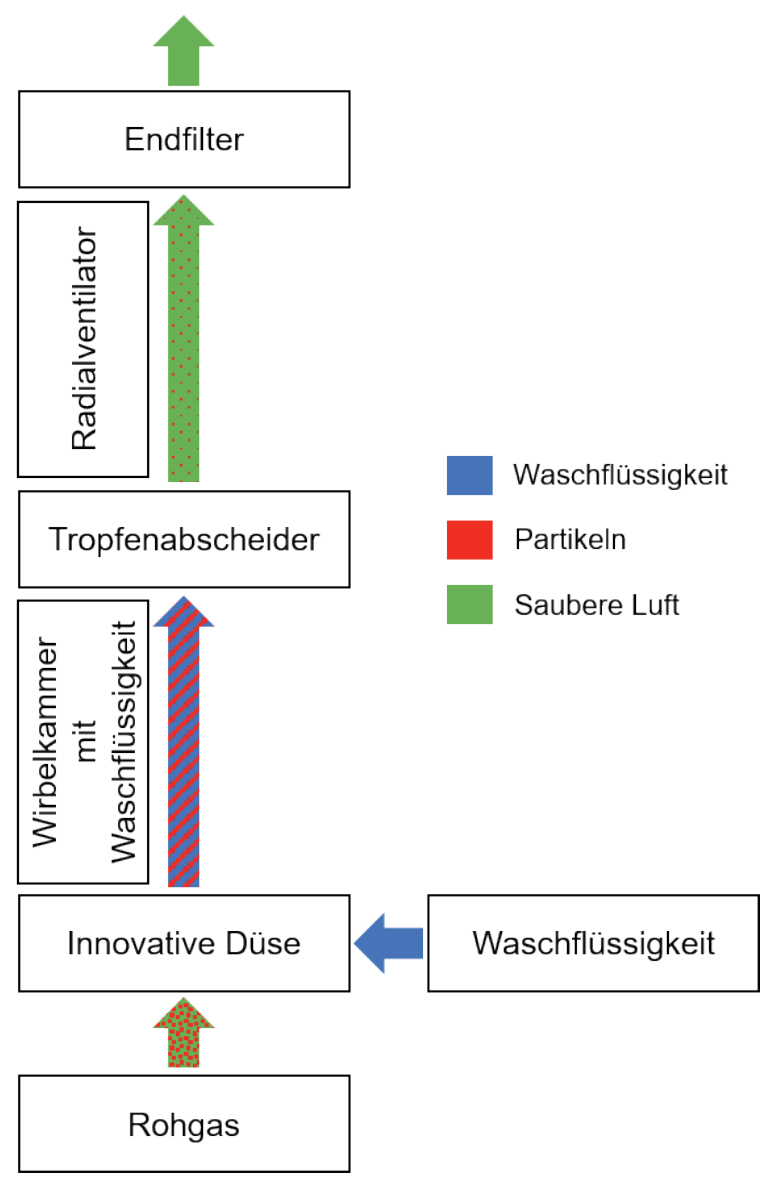

Abbildung 4 - Schematische Darstellung der Versuchsanlage mit abgebildeten Funktionszonen bzw. Komponenten.
Das Rohgas wird mittels eines Radialventilators in eine innovative 3D-gedruckte Düse gefördert. Durch strömungsmechanische Effekte wird innerhalb der Düse die Waschflüssigkeit zum einen hineinbefördert, und zum anderen zerstäubt. Im Anschluss strömt das Aerosol mit dem tropfenförmig vorliegenden Waschwasser in eine Wirbelkammer. In der Wirbelkammer wird zusätzlich Wasser mittels des Luftstroms aufgewirbelt. Durch die Aufwirbelung des Wassers werden weiteren Tropfen gebildet. Aufgrund hoher Differenzgeschwindigkeiten, sowohl in der Düse als auch in der Wirkbelkammer, zwischen den Tropfen und den Partikeln kommt es zum Auftreffen und Haften der Partikeln an der Tropfenoberfläche. Diese Partikel-Tropfen-Agglomerate werden im Anschluss an die Wirbelkammer mittels Tropfenabscheider aus dem Gasstrom auf Basis von Trägheit entfernt. Der nicht am Wasser abgeschiedene Anteil an Partikeln im Gasstrom wird mittels eines Endfilters abgeschieden.

\section{Charakterisierung der Messpositionen}

Bevor die Low-Cost PM-Sensoren (LC-Sensor) in die Versuchsanlage eingebaut werden, müssen zunächst die Messbedingungen bzgl. Temperatur, Strömungsgeschwindigkeiten und relativer Feuchte bestimmt werden. Hierzu soll die Versuchsanlage, ohne die Beaufschlagung von Partikelmaterial, bei verschiedenen Betriebspunkten betrieben werden. Dabei ist die Versuchsanlage zunächst in folgende drei Bereiche eingeteilt worden:

1. Rohgas

2. Tropfenabscheider

3. Endfilter

Diese Bereiche unterscheiden sich im späteren Betrieb wesentlich im Hinblick auf Strömungsgeschwindigkeiten $\mathrm{v}$, relativer Luftfeuchten $\mathrm{rF}$ und konsequenterweise auch in der Partikelanzahl. Die minimalen und maximalen Versuchsbedingungen für die drei Bereiche sind in Tabelle 2 dargestellt. Es fällt auf, dass die verschiedenen Bereiche unterschiedliche Anforderungen an die Partikelmesstechnik stellen. Zum einen, kann es bei hohen relativen Luftfeuchten zum hygroskopischen Wachstum der Partikeln kommen, was zu einer fehlerhaften Konzentrations- und Partikelgrößenmessung führen kann. Dieser Effekt kann durch eine Beheizung der Probenahmestrecke verringert werden. Diese Möglichkeit steht beim LCSensor nicht zur Verfügung. Des Weiteren fallen die unterschiedlichen Strömungsgeschwindigkeiten im Bereich von 0,5 - 35 ms $^{-1}$ auf. Zur Gewährleistung einer isokinetischen Probenahme, kann bei Verwendung des HC-Sensors die Probenahmedüse und somit die Strömungsgeschwindigkeit am Eintritt variiert werden. Dadurch wird eine nichtrepräsentative Probenahme verhindert. Beim LC-Sensor steht diese Variation nicht zur Verfügung. 
Tab. 2: Versuchsbedingungen in den verschiedenen Bereichen der Anlage

\begin{tabular}{|l|c|c|c|}
\hline \multicolumn{1}{|c|}{ Bereich } & Rohgas & $\begin{array}{c}\text { Tropfen- } \\
\text { abscheider }\end{array}$ & Endfilter \\
\hline $\mathrm{v} / \mathrm{ms}^{-1}$ & $9-35$ & $0,58-4,72$ & $0,27-4,06$ \\
\hline $\mathrm{rF} / \%$ & Umgebung & $64,8-88,2$ & $49,4-69,5$ \\
\hline $\mathrm{T} /{ }^{\circ} \mathrm{C}$ & Umgebung & $19,9-22,9$ & $22,2-27,2$ \\
\hline
\end{tabular}

Überprüfung von systematischen Abweichungen zwischen den Sensoren

Zur Überprüfung der systematischen Abweichung der LC-Sensoren werden diese in einer Testkammer mit einem Volumen von $1 \mathrm{~m}^{3}$ unter regulierbaren Feuchtebedingungen mit Partikeln beaufschlagt. Im Rahmen dieser Versuchsreihe werden acht LCSensoren untereinander und zur Referenzmesstechnik (HC-Sensor) verglichen. Hierbei werden sowohl Versuche bei Umgebungsbedingungen bei relativen Feuchten $(\mathrm{rF})$ $<<80 \%$ und bei relativen Feuchten ( $\mathrm{rF}) \quad>80 \%$ durchgeführt. Mittels dieser Vorgehensweise soll ein etwaiger Einfluss der relativen Luftfeuchte auf die Messqualität der LC-Sensoren untersucht werden. Die Partikelzugabe erfolgt mittels Bürstendosierer auf ein Anzahlkonzentrationsniveau von $6000 \mathrm{~cm}^{-3}$. Dies entspricht der zweifachen Messgrenze der Sensoren von Typ 2. Hierbei soll untersucht werden, inwiefern die Sensoren von Typ 2 auch außerhalb der vom Hersteller spezifizierten Messgrenze qualitative Aussagen bzgl. der vorherrschenden Partikelkonzentrationsniveaus treffen können. Bevor die Partikeln in die Versuchskammer gelangen, werden Partikeln >PM2,5 Fraktion abgeschieden. Aufgrund des konstanten Probenahmevolumenstroms des HC-Sensors verhält sich die Konzentrationsabnahme in der Versuchskammer analog zu dem Konzentrationsverlauf in einem idealen Reaktor mit Rührkesselcharakterisitk (CSTR). Mittels des in der Versuchskammer verbauten Ventilators, kann eine ideale Vermischung der Partikeln angenommen und eine Sedimentation ausgeschlossen werden.

\section{Untersuchung der qualitativen Aussagekraft der Messdaten}

Im Anschluss an die systematische Überprüfung der Abweichung zwischen den Sensoren und der Referenzmesstechnik werden diese an den zuvor aus der Charakterisierung der Versuchsanlage bestimmten Messposition verbaut und mit der Referenzmesstechnik abgeglichen. Hierbei sollen Messpositionen identifiziert werden, bei denen die LC-Sensoren zuverlässige Messdaten liefern können.

\section{Zielsetzung}

Mit Hilfe der vorgestellten Methodik soll ein LowCost PM-Sensorencluster entwickelt werden, welches in der Lage ist online Messdaten in ausreichender Qualität bereitzustellen um zu jedem Zeitpunkt eine Aussage bzgl. der vorherrschenden Partikelkonzentrationen im Prozess zu erhalten. Zukünftig kann dieses Cluster das Potential bieten einen adaptiven Abscheideprozess zu entwickeln, welcher mittels der Kenntnis der lokalen Partikelkonzentrationsniveaus in der Lage ist, im energetischen Optimum unter Kenntnis der Abscheideleistung betrieben zu werden.

\section{Literatur}

[1] P. Bächler, J. Meyer, and A. Dittler, "Characterization of the emission behavior of pulse-jet cleaned filters using a low-cost particulate matter sensor/Charakterisierung der Emission von druckstoßgereinigten Oberflächenfiltern mit einem Low-CostFeinstaubsensor," GrdL, vol. 79, 11-12, pp. 443450, 2019, doi: 10.37544/0949-8036-2019-11-12-49.

[2] A. Samad, F. E. Melchor Mimiaga, B. Laquai, and U. Vogt, "Investigating a Low-Cost Dryer Designed for Low-Cost PM Sensors Measuring Ambient Air Quality," Sensors (Basel, Switzerland), vol. 21, no. 3, 2021, doi: 10.3390/s21030804.

[3] E. Weber, "Definition des Benetzungsverhaltens heterogener Stäube und dessen Einfluß auf die Naßentstaubung," [Online]. Available: http://swb.bsz-bw.de/ $\mathrm{DB}=2.1 / \mathrm{PPN}$ ?PPN=1125677511

[4] F. Löffler, Staubabscheiden: 34 Tabellen. Stuttgart: Thieme, 1988.

[5] P. Bächler, J. Szabadi, J. Meyer, and A. Dittler, "Simultaneous measurement of spatially resolved particle emissions in a pilot plant scale baghouse filter applying distributed low-cost particulate matter sensors," Journal of Aerosol Science, vol. 150, p. 105644, 2020, doi: 10.1016/j.jaerosci.2020.105644.

[6] M. Kuhli, Entwicklung von Aufgabesystemen zur Charakterisierung pharmazeutischer Aerosole mit einem optischen Partikelzähler, 2009. [Online]. Available: https://macau.uni-kiel.de/receive/diss_mods_ 00004378

[7] H.-R. Tränkler and E. Obermeier, Eds., Sensortechnik: Handbuch für Praxis und Wissenschaft. Berlin, Heidelberg, s.l.: Springer Berlin Heidelberg, 1998.

[8] M. Stiess, Mechanische Verfahrenstechnik - Partikeltechnologie 1, 3rd ed. Berlin, Heidelberg: Springer Berlin Heidelberg, 2008. [Online]. Available: http:// nbn-resolving.org/urn:nbn:de:bsz:31-epflicht1544055

[9] L. Gail and U. Gommel, Eds., Reinraumtechnik, 4th ed. Berlin, Heidelberg: Springer, 2018. [Online]. Available: http://www.springer.com/ 